SIDOROV M.V.

\title{
GREEN-RVACHEV'S QUASI-FUNCTION METHOD FOR CONSTRUCTING TWO-SIDED APPROXIMATIONS TO POSITIVE SOLUTION OF NONLINEAR BOUNDARY VALUE PROBLEMS
}

\begin{abstract}
A homogeneous Dirichlet problem for a semilinear elliptic equations with the Laplace operator and Helmholtz operator is investigated. To construct the two-sided approximations to a positive solution of this boundary value problem the transition to an equivalent nonlinear integral equation (with the help of the Green-Rvachev's quasi-function) with its subsequent analysis by methods of the theory of semi-ordered spaces is used. The work and efficiency of the developed method are demonstrated by a computational experiment for a test problem with exponential nonlinearity.

Key words and phrases: positive solution, semilinear elliptic boundary value problem, heterotone operator, two-sided approach, Green-Rvachev's quasi-function.
\end{abstract}

Kharkiv National University of Radio Electronics, 14 Nauki ave., 61166, Kharkiv, Ukraine

E-mail: maxim.sidorov@nure.ua

\section{INTRODUCTION}

Let us consider the problem of finding a positive solution of a semilinear elliptic equation with a homogeneous Dirichlet condition:

$$
\begin{gathered}
\mathfrak{L} u=f(\mathbf{x}, u), \quad \mathbf{x} \in \Omega, \\
u(\mathbf{x})>0, \quad \mathbf{x} \in \Omega, \\
\left.u\right|_{\partial \Omega}=0,
\end{gathered}
$$

where $\mathfrak{L} u \equiv-\Delta u$ or $\mathfrak{L} u \equiv-\Delta u+\kappa^{2} u, \Omega$ is a bounded Jordan-measurable domain from $\mathbb{R}^{2}$ or $\mathbb{R}^{3}$ with piecewise smooth boundary $\partial \Omega(\bar{\Omega}=\Omega \cup \partial \Omega), \Delta$ is the Laplace operator, $\mathbf{x}=\left(x_{1}, x_{2}\right)$, $\Delta=\frac{\partial^{2}}{\partial x_{1}^{2}}+\frac{\partial^{2}}{\partial x_{2}^{2}}$, if $\Omega \subset \mathbb{R}^{2}$, and $\mathbf{x}=\left(x_{1}, x_{2}, x_{3}\right), \Delta=\frac{\partial^{2}}{\partial x_{1}^{2}}+\frac{\partial^{2}}{\partial x_{2}^{2}}+\frac{\partial^{2}}{\partial x_{3}^{2}}$, if $\Omega \subset \mathbb{R}^{3}$.

Let us assume that the function $f(\mathbf{x}, u)$ is continuous and positive for $\mathbf{x} \in \bar{\Omega}, u>0$.

The problem (1)-(3) is often encountered in the mathematical modeling of nonlinear stationary processes considered in thermophysics, electromagnetism, biology, chemical kinetics, etc. [11]. In this case, the condition of positivity (2) naturally arises from the meaning of the function $u$ in a particular applied field. It is convenient to carry out the analysis of the problem by the methods of the theory of nonlinear operators in semi-ordered spaces $[1,5,9,10]$, passing to the equivalent Hammerstein integral equation with the help of the Green's functions method. In this case, it is possible to construct a two-sided iteration process to the desired solution $[4,14]$. But, the practical application of this approach has certain limitations due to 
the fact that the analytic expression of the Green's function must be known. It is possible to get rid of this restriction, if the corresponding Green-Rvachev's quasi-function [12,14] will be used instead of Green's function.

The purpose of the paper is to develop the iterative methods for solving the boundary value problem (1)-(3), which have a two-sided nature of convergence to the desired solution and are not tied to the presence of a known Green's function. Two-sided approximate methods for solving nonlinear operator equations based on the theory of nonlinear operators in semiordered spaces were developed in $[3,6,13$, etc.]. This paper continues the research begun in $[4,14]$, and extends them to the areas of arbitrary geometry and elliptic equations with the Helmholtz operator.

\section{CONSTRUCTION OF AN EQUIVALENT INTEGRAL EQUATION}

To construct an integral equation that is equivalent to the problem (1)-(3), let us use the Green-Rvachev's quasi-function $[12,14]$.

Let the boundary $\partial \Omega$ of the domain $\Omega$ consists of a finite number of pieces of lines $\sigma_{i}(\mathbf{x})=0$, $i=1,2, \ldots, r$, where each $\sigma_{i}(\mathbf{x})$ is an elementary function. Then with the help of the Rfunctions method [12] one can construct in the form of a single analytic expression an elementary function $\omega(\mathbf{x})$, which describes the geometry of the domain $\Omega$, that is:

a) $\omega(\mathbf{x})>0$ in $\Omega$;

b) $\omega(\mathbf{x})=0$ on $\partial \Omega$;

c) $|\nabla \omega(\mathbf{x})| \neq 0$ on $\partial \Omega$.

Also, the function $\omega(\mathbf{x})$ can have certain properties of differentiation due to the use of various sufficiently complete systems of R-functions [12].

Definition 1. Let $g_{m}(r)$ be a fundamental solution of the equation $\mathfrak{L} u=0$ in $\mathbb{R}^{m}$. The GreenRvachev's quasi-function of the first boundary value problem for the operator $\mathfrak{L}$ in $\mathbb{R}^{m}$ is the function

$$
\begin{gathered}
Q_{m}(\mathbf{x}, \mathbf{s})=g_{m}(r)-\tilde{g}_{m}(\mathbf{x}, \mathbf{s}), \\
\text { where } \mathbf{x}=\left(x_{1}, \ldots, x_{m}\right), \mathbf{s}=\left(s_{1}, \ldots, s_{m}\right), r=|\mathbf{x}-\mathbf{s}|=\sqrt{\sum_{i=1}^{m}\left(x_{i}-s_{i}\right)^{2}}, \\
\tilde{g}_{m}(\mathbf{x}, \mathbf{s})=g_{m}\left(\sqrt{r^{2}+4 \omega(\mathbf{x}) \omega(\mathbf{s})}\right),
\end{gathered}
$$

$\omega(\mathbf{x})$ is the function that describes the geometry of the domain $\Omega$.

Let us note [12] that for the case when $\mathfrak{L} u \equiv-\Delta u, \Omega$ is a ball of radius $R$ in $\mathbb{R}^{m}$, and $\omega(\mathbf{x})=\frac{1}{2 R}\left(R^{2}-x_{1}^{2}-\cdots-x_{m}^{2}\right)$, the Green-Rvachev's quasi-function (4) turns into the exact Green's function of the first boundary value problem for the Laplace operator considered in a ball $\Omega$.

The fundamental solutions of the Laplace equation $-\Delta u=0$ in $\mathbb{R}^{2}$ and $\mathbb{R}^{3}$ have the form

$$
g_{2}(r)=\frac{1}{2 \pi} \ln \frac{1}{r}, \quad g_{3}(r)=\frac{1}{4 \pi} \cdot \frac{1}{r}
$$


consequently, the Green-Rvachev's quasi-function of the first boundary value problem for the operator $-\Delta$ acquires the form

$$
\begin{gathered}
Q_{2}(\mathbf{x}, \mathbf{s})=\frac{1}{2 \pi} \ln \sqrt{1+\frac{4 \omega(\mathbf{x}) \omega(\mathbf{s})}{r^{2}}} \text { in } \mathbb{R}^{2}, \\
Q_{3}(\mathbf{x}, \mathbf{s})=\frac{1}{4 \pi} \cdot \frac{\sqrt{r^{2}+4 \omega(\mathbf{x}) \omega(\mathbf{s})}-r}{r \sqrt{r^{2}+4 \omega(\mathbf{x}) \omega(\mathbf{s})}} \text { in } \mathbb{R}^{3} .
\end{gathered}
$$

For the Helmholtz equation $-\Delta u+\kappa^{2} u=0$ in $\mathbb{R}^{2}$ and $\mathbb{R}^{3}$ the fundamental solutions have the form

$$
g_{2}(r)=\frac{1}{2 \pi} K_{0}(\kappa r), \quad g_{3}(r)=\frac{1}{4 \pi r} e^{-\kappa r},
$$

where $K_{0}(z)$ is modified Bessel function of the second kind, and the Green-Rvachev's quasifunction of the first boundary value problem for the operator $-\Delta+\kappa^{2}$ acquires the form

$$
\begin{gathered}
Q_{2}(\mathbf{x}, \mathbf{s})=\frac{1}{2 \pi}\left(K_{0}(\kappa r)-K_{0}\left(\kappa \sqrt{r^{2}+4 \omega(\mathbf{x}) \omega(\mathbf{s})}\right)\right) \text { in } \mathbb{R}^{2}, \\
Q_{3}(\mathbf{x}, \mathbf{s})=\frac{1}{4 \pi} \cdot \frac{\sqrt{r^{2}+4 \omega(\mathbf{x}) \omega(\mathbf{s})} e^{-\kappa r}-r e^{-\kappa \sqrt{r^{2}+4 \omega(\mathbf{x}) \omega(\mathbf{s})}}}{r \sqrt{r^{2}+4 \omega(\mathbf{x}) \omega(\mathbf{s})}} \text { in } \mathbb{R}^{3} .
\end{gathered}
$$

From (5)-(8) and Definition 1 the following lemma on the properties of the Green-Rvachev's quasi-function follows.

Lemma 1. The Green-Rvachev's quasi-function (4) has the following properties:

a) $Q_{m}(\mathbf{x}, \mathbf{s})=0$ on $\partial \Omega$;

b) is a symmetric function: $Q_{m}(\mathbf{x}, \mathbf{s})=Q_{m}(\mathbf{s}, \mathbf{x})$;

c) has the same feature for $\mathbf{x}=\mathbf{s}$ as the usual Green's function;

d) is positive in the domain $\Omega: Q_{m}(\mathbf{x}, \mathbf{s})>0, \mathbf{x}, \mathbf{s} \in \Omega, \mathbf{x} \neq \mathbf{s}$.

For the function $u \in C^{2}(\Omega) \cap C^{1}(\bar{\Omega})$ such that $\mathfrak{L} u \in L_{2}(\Omega)$, the following integral representation $[7,8]$ holds:

$$
u(\mathbf{x})=\int_{\partial \Omega}\left[g(\mathbf{x}, \mathbf{s}) \frac{\partial u(\mathbf{s})}{\partial \mathbf{n}_{\mathbf{s}}}-u(\mathbf{s}) \frac{\partial g(\mathbf{x}, \mathbf{s})}{\partial \mathbf{n}_{\mathbf{s}}}\right] d_{\mathbf{s}} \sigma+\int_{\Omega} g(\mathbf{x}, \mathbf{s}) \mathfrak{L}_{\mathbf{s}} u(\mathbf{s}) d \mathbf{s}, \quad \mathbf{x} \in \Omega,
$$

and for the functions $u, \tilde{g} \in C^{2}(\bar{\Omega})$ the second Green's formula [7]

$$
0=-\int_{\Omega}\left[\tilde{g}(\mathbf{s}) \mathfrak{L}_{\mathbf{s}} u(\mathbf{s})-u(\mathbf{s}) \mathfrak{L}_{\mathbf{s}} \tilde{g}(\mathbf{s})\right] d \mathbf{s}+\int_{\partial \Omega}\left[u(\mathbf{s}) \frac{\partial \tilde{g}(\mathbf{s})}{\partial \mathbf{n}_{\mathbf{s}}}-\tilde{g}(\mathbf{s}) \frac{\partial u(\mathbf{s})}{\partial \mathbf{n}_{\mathbf{s}}}\right] d_{\mathbf{s}} \sigma
$$

holds.

In formulas (9), (10) $\mathbf{n}_{\mathbf{s}}$ is the outer to $\partial \Omega$ normal in the variables $\mathbf{s}, d_{\mathbf{s}} \sigma$ means that the integration for $\mathbf{s}$ is along $\partial \Omega, \mathfrak{L}_{\mathrm{s}} u \equiv-\Delta_{\mathrm{s}} u$ or $\mathfrak{L}_{\mathrm{s}} u \equiv-\Delta_{\mathrm{s}} u+\kappa^{2} u, \Delta_{\mathrm{s}}=\frac{\partial^{2}}{\partial s_{1}^{2}}+\frac{\partial^{2}}{\partial s_{2}^{2}}$, if $\Omega \subset \mathbb{R}^{2}$, and $\Delta_{\mathbf{s}}=\frac{\partial^{2}}{\partial s_{1}^{2}}+\frac{\partial^{2}}{\partial s_{2}^{2}}+\frac{\partial^{2}}{\partial s_{3}^{2}}$, if $\Omega \subset \mathbb{R}^{3}$. 
Let $u$ be a classical solution of the problem (1)-(3), and let us choose the function $\tilde{g}$ in (10) as in Definition 1. Adding the equalities (9) and (10), taking into account (4), we obtain

$$
\begin{aligned}
u(\mathbf{x}) & =\int_{\Omega} \mathfrak{L}_{\mathbf{s}} \tilde{g}(\mathbf{x}, \mathbf{s}) \cdot u(\mathbf{s}) d \mathbf{s}+\int_{\Omega} Q_{m}(\mathbf{x}, \mathbf{s}) \cdot \mathfrak{L}_{\mathbf{s}} u(\mathbf{s}) d \mathbf{s} \\
& +\int_{\partial \Omega}\left[Q_{m}(\mathbf{x}, \mathbf{s}) \frac{\partial u(\mathbf{s})}{\partial \mathbf{n}_{\mathbf{s}}}-u(\mathbf{s}) \frac{\partial Q_{m}(\mathbf{x}, \mathbf{s})}{\partial \mathbf{n}_{\mathbf{s}}}\right] d_{\mathbf{s}} \sigma .
\end{aligned}
$$

Then, taking into account that $Q_{m}(\mathbf{x}, \mathbf{s})=0$ and $u(\mathbf{x})=0$ on $\partial \Omega$, and making allowance for the equation (1), we finally obtain the integral equation for the function $u$ in the form

$$
u(\mathbf{x})=\int_{\Omega} K_{m}(\mathbf{x}, \mathbf{s}) u(\mathbf{s}) d \mathbf{s}+\int_{\Omega} Q_{m}(\mathbf{x}, \mathbf{s}) f(\mathbf{s}, u(\mathbf{s})) d \mathbf{s},
$$

where $K_{m}(\mathbf{x}, \mathbf{s})=\mathfrak{L}_{\mathbf{s}} \tilde{g}(\mathbf{x}, \mathbf{s})$.

The nonlinear integral equation (11) can be written in the form of Urysohn equation

$$
u(\mathbf{x})=\int_{\Omega} P(\mathbf{x}, \mathbf{s}, u(\mathbf{s})) d \mathbf{s},
$$

where $P(\mathbf{x}, \mathbf{s}, u(\mathbf{s}))=K_{m}(\mathbf{x}, \mathbf{s}) u(\mathbf{s})+Q_{m}(\mathbf{x}, \mathbf{s}) f(\mathbf{s}, u(\mathbf{s}))$.

If the boundary value problem (1)-(3) has a classical solution, then it also satisfies the equation (11). If the classical solution of the problem does not exist, then the equation (11) can be used to introduce the concept of a generalized solution of the boundary value problem (1)-(3).

The equation (11) will be considered in a Banach space $C(\bar{\Omega})$ of the functions continuous in $\bar{\Omega}$. The norm in $C(\bar{\Omega})$ is entered by the rule $\|u\|=\max _{\mathbf{x} \in \bar{\Omega}}|u(\mathbf{x})|$. Let us select in $C(\bar{\Omega})$ the cone $\mathcal{K}_{+}=\{u \in C(\bar{\Omega}): u(\mathbf{x}) \geq 0, \mathbf{x} \in \bar{\Omega}\}$ of non-negative functions. Note that the cone $\mathcal{K}_{+}$ in $C(\bar{\Omega})$ is normal (and even acute). With the help of the cone $\mathcal{K}_{+}$in the space $C(\bar{\Omega})$ let us introduce a semiordering by the rule:

$$
\text { for } u, v \in C(\bar{\Omega}) \quad u \leqslant v, \text { if } v-u \in \mathcal{K}_{+},
$$

that is,

$$
u \leqslant v, \quad \text { if } u(\mathbf{x}) \leq v(\mathbf{x}) \text { for all } \mathbf{x} \in \bar{\Omega} .
$$

Definition 2. By a solution (generalized) of the boundary value problem (1)-(3) will be meant a function $u^{*} \in \mathcal{K}_{+}$, which is a solution of the integral equation (11).

\section{CONSTRUCTION OF A PROCESS OF TWO-SIDED APPROXIMATIONS}

Let us construct a process of two-sided approximations for finding the solution of the integral equation (11) (and consequently, the solution of the boundary value problem (1)-(3) using the methods of the theory of nonlinear operators in semi-ordered spaces $[1,5,9,10]$.

Let us introduce a nonlinear operator $T$ acting in $C(\bar{\Omega})$ by the rule

$$
T(u)(\mathbf{x})=\int_{\Omega} P(\mathbf{x}, \mathbf{s}, u(\mathbf{s})) d \mathbf{s} .
$$


Let us denote

$$
K_{m}^{+}(\mathbf{x}, \mathbf{s})=\max \left\{0, K_{m}(\mathbf{x}, \mathbf{s})\right\}, \quad K_{m}^{-}(\mathbf{x}, \mathbf{s})=\max \left\{0,-K_{m}(\mathbf{x}, \mathbf{s})\right\} .
$$

Then $K_{m}^{+}(\mathbf{x}, \mathbf{s}) \geq 0, K_{m}^{-}(\mathbf{x}, \mathbf{s}) \geq 0$ for $\mathbf{x}, \mathbf{s} \in \Omega(\mathbf{x} \neq \mathbf{s})$,

$$
K_{m}(\mathbf{x}, \mathbf{s})=K_{m}^{+}(\mathbf{x}, \mathbf{s})-K_{m}^{-}(\mathbf{x}, \mathbf{s}), \quad\left|K_{m}(\mathbf{x}, \mathbf{s})\right|=K_{m}^{+}(\mathbf{x}, \mathbf{s})+K_{m}^{-}(\mathbf{x}, \mathbf{s}),
$$

and operator $T$ of the form (12) will be written in the form

$$
T(u)(\mathbf{x})=\int_{\Omega} K_{m}^{+}(\mathbf{x}, \mathbf{s}) u(\mathbf{s}) d \mathbf{s}-\int_{\Omega} K_{m}^{-}(\mathbf{x}, \mathbf{s}) u(\mathbf{s}) d \mathbf{s}+\int_{\Omega} Q_{m}(\mathbf{x}, \mathbf{s}) f(\mathbf{s}, u(\mathbf{s})) d \mathbf{s} .
$$

Suppose that the function $f(\mathbf{x}, u)$ allows a diagonal representation $f(\mathbf{x}, u)=\hat{f}(\mathbf{x}, u, u)$, besides, continuous on the sets of variables $\mathbf{x}, v, w$ non-negative function $\hat{f}(\mathbf{x}, v, w)$ monotonically increases with respect to $v$ and monotonically decreases with respect to $w$ for all $\mathbf{x} \in \Omega$. Then the operator $T$ of the form (13) will be heterotone with the companion operator

$$
\hat{T}(v, w)(\mathbf{x})=\int_{\Omega} K_{m}^{+}(\mathbf{x}, \mathbf{s}) v(\mathbf{s}) d \mathbf{s}-\int_{\Omega} K_{m}^{-}(\mathbf{x}, \mathbf{s}) w(\mathbf{s}) d \mathbf{s}+\int_{\Omega} Q_{m}(\mathbf{x}, \mathbf{s}) \hat{f}(\mathbf{s}, v(\mathbf{s}), w(\mathbf{s})) d \mathbf{s} .
$$

Operators $T$ and $\hat{T}$ are completely continuous.

Note that for the case when the function $f(\mathbf{x}, u)$ increases monotonically with respect to $u$ for all $\mathbf{x} \in \Omega$ we can choose $\hat{f}(\mathbf{x}, v, w)=f(\mathbf{x}, v)$, and if it decreases monotonically with respect to $u$ for all $\mathbf{x} \in \Omega$ we can set $\hat{f}(\mathbf{x}, v, w)=f(\mathbf{x}, w)$.

In the cone $\mathcal{K}_{+}$let us select a strongly invariant cone segment $\left\langle v^{0}, w^{0}\right\rangle$ by conditions $\hat{T}\left(v^{0}, w^{0}\right) \geq v^{0}, \hat{T}\left(w^{0}, v^{0}\right) \leq w^{0}$, which for the operator $\hat{T}$ that is defined by (14) will have the form:

$$
\begin{aligned}
& \int_{\Omega} K_{m}^{+}(\mathbf{x}, \mathbf{s}) v^{0}(\mathbf{s}) d \mathbf{s}-\int_{\Omega} K_{m}^{-}(\mathbf{x}, \mathbf{s}) w^{0}(\mathbf{s}) d \mathbf{s} \\
& +\int_{\Omega} Q_{m}(\mathbf{x}, \mathbf{s}) \hat{f}\left(\mathbf{s}, v^{0}(\mathbf{s}), w^{0}(\mathbf{s})\right) d \mathbf{s} \geq v^{0}(\mathbf{x}) \text { for all } \mathbf{x} \in \bar{\Omega}, \\
& \int_{\Omega} K_{m}^{+}(\mathbf{x}, \mathbf{s}) w^{0}(\mathbf{s}) d \mathbf{s}-\int_{\Omega} K_{m}^{-}(\mathbf{x}, \mathbf{s}) v^{0}(\mathbf{s}) d \mathbf{s} \\
& +\int_{\Omega} Q_{m}(\mathbf{x}, \mathbf{s}) \hat{f}\left(\mathbf{s}, w^{0}(\mathbf{s}), v^{0}(\mathbf{s})\right) d \mathbf{s} \leq w^{0}(\mathbf{x}) \text { for all } \mathbf{x} \in \bar{\Omega} .
\end{aligned}
$$

Let us form an iterative process by the scheme $v^{(k+1)}=\hat{T}\left(v^{(k)}, w^{(k)}\right), w^{(k+1)}=\hat{T}\left(w^{(k)}, v^{(k)}\right)$, $k=0,1,2, \ldots\left(v^{(0)}=v^{0}, w^{(0)}=w^{0}\right)$ :

$$
\begin{aligned}
v^{(k+1)}(\mathbf{x}) & =\int_{\Omega} K_{m}^{+}(\mathbf{x}, \mathbf{s}) v^{(k)}(\mathbf{s}) d \mathbf{s}-\int_{\Omega} K_{m}^{-}(\mathbf{x}, \mathbf{s}) w^{(k)}(\mathbf{s}) d \mathbf{s} \\
& +\int_{\Omega} Q_{m}(\mathbf{x}, \mathbf{s}) \hat{f}\left(\mathbf{s}, v^{(k)}(\mathbf{s}), w^{(k)}(\mathbf{s})\right) d \mathbf{s}, \quad k=0,1,2, \ldots,
\end{aligned}
$$




$$
\begin{aligned}
w^{(k+1)}(\mathbf{x})= & \int_{\Omega} K_{m}^{+}(\mathbf{x}, \mathbf{s}) w^{(k)}(\mathbf{s}) d \mathbf{s}-\int_{\Omega} K_{m}^{-}(\mathbf{x}, \mathbf{s}) v^{(k)}(\mathbf{s}) d \mathbf{s} \\
+ & \int_{\Omega} Q_{m}(\mathbf{x}, \mathbf{s}) \hat{f}\left(\mathbf{s}, w^{(k)}(\mathbf{s}), v^{(k)}(\mathbf{s})\right) d \mathbf{s}, \quad k=0,1,2, \ldots, \\
& v^{(0)}(\mathbf{x})=v^{0}(\mathbf{x}), \quad w^{(0)}(\mathbf{x})=w^{0}(\mathbf{x}) .
\end{aligned}
$$

Since the cone segment $\left\langle v^{0}, w^{0}>\right.$ is strongly invariant for the heterotone operator $T$ for which the operator $\hat{T}$ is a companion one, it follows that the sequence $\left\{v^{(k)}(\mathbf{x})\right\}$ does not decrease with respect to the cone $\mathcal{K}_{+}$, and the sequence $\left\{w^{(k)}(\mathbf{x})\right\}$ does not increase with respect to the cone $\mathcal{K}_{+}$. Besides, the cone $\mathcal{K}_{+}$is normal and the operator $\hat{T}$ is completely continuous, so the boundaries $v^{*}(\mathbf{x})$ and $w^{*}(\mathbf{x})$ of these sequences exist. Thus, the chain of inequalities holds:

$$
v^{0}=v^{(0)} \leqslant v^{(1)} \leqslant \cdots \leqslant v^{(k)} \leqslant \cdots \leqslant v^{*} \leqslant w^{*} \leqslant \cdots \leqslant w^{(k)} \leqslant \cdots \leqslant w^{(1)} \leqslant w^{(0)}=w^{0} .
$$

There are two possible cases: $v^{*}<w^{*}$ and $v^{*}=w^{*}$. In the second case, $u^{*}:=v^{*}=w^{*}$ is the unique on $\left\langle v^{0}, w^{0}>\right.$ fixed point of the operator $T$, that is, it is the unique on $\left\langle v^{0}, w^{0}\right\rangle$ solution of the boundary value problem (1)-(3).

The functions $v^{*}(\mathbf{x})$ and $w^{*}(\mathbf{x})$ are a solution of the system of equations $v=\hat{T}(v, w)$, $w=\hat{T}(w, v)$, which in the considered case has the form

$$
\begin{aligned}
& v(\mathbf{x})=\int_{\Omega} K_{m}^{+}(\mathbf{x}, \mathbf{s}) v(\mathbf{s}) d \mathbf{s}-\int_{\Omega} K_{m}^{-}(\mathbf{x}, \mathbf{s}) w(\mathbf{s}) d \mathbf{s}+\int_{\Omega} Q_{m}(\mathbf{x}, \mathbf{s}) \hat{f}(\mathbf{s}, v(\mathbf{s}), w(\mathbf{s})) d \mathbf{s}, \\
& w(\mathbf{x})=\int_{\Omega} K_{m}^{+}(\mathbf{x}, \mathbf{s}) w(\mathbf{s}) d \mathbf{s}-\int_{\Omega} K_{m}^{-}(\mathbf{x}, \mathbf{s}) v(\mathbf{s}) d \mathbf{s}+\int_{\Omega} Q_{m}(\mathbf{x}, \mathbf{s}) \hat{f}(\mathbf{s}, w(\mathbf{s}), v(\mathbf{s})) d \mathbf{s} .
\end{aligned}
$$

The equality $v^{*}=w^{*}$ will hold if the system (20), (21) does not have on $\left\langle v^{0}, w^{0}>\right.$ such solutions that $v \neq w[9,10]$.

Thus, such a theorem is true.

Theorem 1. Let $\left\langle v^{0}, w^{0}>\right.$ be a strongly invariant cone segment for the heterotone operator $T$ of the form (13) with the companion operator $\hat{T}$ of the form (14) and the system of equations (20), (21) does not have on $\left\langle v^{0}, w^{0}>\right.$ solutions such that $v \neq w$. Then the iterative process (17)-(19) converges in the norm of the space $C(\bar{\Omega})$ to the unique on $\left\langle v^{0}, w^{0}\right\rangle$ continuous positive solution $u^{*}$ of the boundary value problem (1)-(3), and a chain of inequalities holds:

$$
v^{0}=v^{(0)} \leqslant v^{(1)} \leqslant \cdots \leqslant v^{(k)} \leqslant \cdots \leqslant u^{*} \leqslant \cdots \leqslant w^{(k)} \leqslant \cdots \leqslant w^{(1)} \leqslant w^{(0)}=w^{0} .
$$

Note that the chain of inequalities (22) characterizes the iterative process (17)-(19) as a method of two-sided approximations.

From the chain of inequalities (22) it follows that each of the cone segments $\left\langle v^{(k)}, w^{(k)}\right\rangle$, $k=0,1,2, \ldots$, is strongly invariant for the heterotone operator $T$ of the form (13) with the companion operator $\hat{T}$ of the form (14).

Let us determine the conditions for the existence of a unique positive solution of the boundary value problem (1)-(3) and two-sided convergence of the successive approximations (17)-(19) to it, by clarifying the conditions under which the system of equations (20), (21) does 
not have on some of the strongly invariant cone segments $\left\langle v^{(k)}, w^{(k)}>, k=0,1,2, \ldots\right.$, such solutions that $v \neq w$.

First we use such a condition $[9,10]$ : if

$$
\hat{T}(v+u, w-u) \ngtr \hat{T}(v, w)+u,
$$

where $u>\theta, v<w, v, w, v+u, w-u \in<v^{0}, w^{0}>$, then the system $v=\hat{T}(v, w), w=\hat{T}(w, v)$ does not have solutions on $\left\langle v^{0}, w^{0}>\right.$, such that $v \neq w$.

Theorem 2. Let $\left\langle v^{0}, w^{0}\right\rangle$ be a strongly invariant cone segment for the heterotone operator $T$ of the form (13) with the companion operator $\hat{T}$ of the form (14) and the following condition holds: for any numbers $v, w, u$ such, that $0<v<w, 0<u<w$, and for all $\mathbf{x} \in \Omega$ the following inequality is satisfied:

$$
\hat{f}(\mathbf{x}, v+u, w-u)<\hat{f}(\mathbf{x}, v, w)+\frac{u}{M+M_{1}}
$$

where

$$
M=\max _{\mathbf{x} \in \bar{\Omega}} \int_{\Omega} Q_{m}(\mathbf{x}, \mathbf{s}) d \mathbf{s}, \quad M_{1}=\max _{\mathbf{x} \in \bar{\Omega}} \int_{\Omega}\left[K_{m}^{+}(\mathbf{x}, \mathbf{s})+K_{m}^{-}(\mathbf{x}, \mathbf{s})\right] d \mathbf{s} .
$$

Then, the iterative process (17)-(19) bilaterally converges in the norm of the space $C(\bar{\Omega})$ to the unique on $\left\langle v^{0}, w^{0}>\right.$ continuous positive solution $u^{*}$ of the boundary value problem $(1)-(3)$.

Proof. Let $u$ and $w-v$ be such functions from $\mathcal{K}_{+} \backslash\{\theta\}(\theta$ is a zero element of $C(\bar{\Omega})$ ), that $v, w, v+u, w-u \in<v^{0}, w^{0}>$. Then $u(\mathbf{x}) \geq 0$ in $\bar{\Omega}$ and $\left.u\right|_{\partial \Omega}=0$. So, if the function $u(\mathbf{x})$ gets the maximum value at the point $\mathbf{x}_{0}$, then $\mathbf{x}_{0} \in \Omega$. Thus,

$$
\begin{aligned}
& \hat{T}(v+u, w-u)\left(\mathbf{x}_{0}\right) \\
& =\int_{\Omega} K_{m}^{+}\left(\mathbf{x}_{0}, \mathbf{s}\right)[v(\mathbf{s})+u(\mathbf{s})] d \mathbf{s}-\int_{\Omega} K_{m}^{-}\left(\mathbf{x}_{0}, \mathbf{s}\right)[w(\mathbf{s})-u(\mathbf{s})] d \mathbf{s} \\
& +\int_{\Omega} Q_{m}\left(\mathbf{x}_{0}, \mathbf{s}\right) \hat{f}(\mathbf{x}, v(\mathbf{s})+u(\mathbf{s}), w(\mathbf{s})-u(\mathbf{s})) d \mathbf{s} \\
& <\int_{\Omega} K_{m}^{+}\left(\mathbf{x}_{0}, \mathbf{s}\right) v(\mathbf{s}) d \mathbf{s}-\int_{\Omega} K_{m}^{-}\left(\mathbf{x}_{0}, \mathbf{s}\right) w(\mathbf{s}) d \mathbf{s}+\int_{\Omega}\left[K_{m}^{+}\left(\mathbf{x}_{0}, \mathbf{s}\right)+K_{m}^{-}\left(\mathbf{x}_{0}, \mathbf{s}\right)\right] u(\mathbf{s}) d \mathbf{s} \\
& +\int_{\Omega} Q_{m}\left(\mathbf{x}_{0}, \mathbf{s}\right)\left[\hat{f}(\mathbf{s}, v(\mathbf{s}), w(\mathbf{s}))+\frac{u(\mathbf{s})}{M+M_{1}}\right] d \mathbf{s} \\
& \leq \int_{\Omega} K_{m}^{+}\left(\mathbf{x}_{0}, \mathbf{s}\right) v(\mathbf{s}) d \mathbf{s}-\int_{\Omega} K_{m}^{-}\left(\mathbf{x}_{0}, \mathbf{s}\right) w(\mathbf{s}) d \mathbf{s}+\int_{\Omega} Q_{m}\left(\mathbf{x}_{0}, \mathbf{s}\right) \hat{f}(\mathbf{s}, v(\mathbf{s}), w(\mathbf{s})) d \mathbf{s} \\
& +\frac{1}{M+M_{1} \mathbf{x}_{\mathbf{n}} \bar{\Omega}} u(\mathbf{x})\left[\int_{\Omega}\left[K_{m}^{+}\left(\mathbf{x}_{0}, \mathbf{s}\right)+K_{m}^{-}\left(\mathbf{x}_{0}, \mathbf{s}\right)\right] d \mathbf{s}+\int_{\Omega} Q_{m}\left(\mathbf{x}_{0}, \mathbf{s}\right) d \mathbf{s}\right] \\
& \leq \int_{\Omega} K_{m}^{+}\left(\mathbf{x}_{0}, \mathbf{s}\right) v(\mathbf{s}) d \mathbf{s}-\int_{\Omega} K_{m}^{-}\left(\mathbf{x}_{0}, \mathbf{s}\right) w(\mathbf{s}) d \mathbf{s}+\int_{\Omega} Q_{m}\left(\mathbf{x}_{0}, \mathbf{s}\right) \hat{f}(\mathbf{s}, v(\mathbf{s}), w(\mathbf{s})) d \mathbf{s}+u\left(\mathbf{x}_{0}\right) \\
& =\hat{T}(v, w)\left(\mathbf{x}_{0}\right)+u\left(\mathbf{x}_{0}\right),
\end{aligned}
$$

that is, the condition (23) holds, consequently, the system of equations (20), (21) does not have on $\left\langle v^{0}, w^{0}>\right.$ such solutions that $v \neq w$ and the Theorem 1 is valid. 
Another condition that ensures equality $v^{*}=w^{*}$ is the existence of $\gamma \in(0 ; 1)$ such that

$$
\|\hat{T}(v, w)-\hat{T}(w, v)\| \leq \gamma\|v-w\|
$$

for all $v, w \in<v^{0}, w^{0}>[2]$.

Let there exists a number $L>0$, that the function $\hat{f}(\mathbf{x}, v, w)$ for all numbers $v, w$ such that $0<v, w<M_{0}$, where $M_{0}=\max _{\mathbf{x} \in \bar{\Omega}} w^{0}(\mathbf{x})$, and for all $\mathbf{x} \in \Omega$ satisfies the inequality

$$
|\hat{f}(\mathbf{x}, w, v)-\hat{f}(\mathbf{x}, v, w)| \leq L|w-v| .
$$

Let us consider the difference $\hat{T}(v, w)(\mathbf{x})-\hat{T}(w, v)(\mathbf{x})$ :

$$
\begin{aligned}
\hat{T}(w, v)(\mathbf{x})-\hat{T}(v, w)(\mathbf{x}) & =\int_{\Omega}\left[K_{m}^{+}(\mathbf{x}, \mathbf{s})+K_{m}^{-}(\mathbf{x}, \mathbf{s})\right][w(\mathbf{s})-v(\mathbf{s})] d \mathbf{s} \\
& +\int_{\Omega} Q_{m}(\mathbf{x}, \mathbf{s})[\hat{f}(\mathbf{s}, w(\mathbf{s}), v(\mathbf{s}))-\hat{f}(\mathbf{s}, v(\mathbf{s}), w(\mathbf{s}))] d \mathbf{s} .
\end{aligned}
$$

Then, taking into account the inequality (25), we obtain an estimate

$$
\begin{aligned}
\|\hat{T}(w, v)-\hat{T}(v, w)\| & =\max _{\mathbf{x} \in \bar{\Omega}}|\hat{T}(w, v)(\mathbf{x})-\hat{T}(v, w)(\mathbf{x})| \\
& \leq\left(M_{1}+L M\right) \cdot \max _{\mathbf{x} \in \bar{\Omega}}|w(\mathbf{x})-v(\mathbf{x})|=\left(M_{1}+L M\right)\|w-v\|,
\end{aligned}
$$

where constants $M$ and $M_{1}$ are defined by equalities (24).

Thus,

$$
\|\hat{T}(w, v)-\hat{T}(v, w)\| \leq \gamma\|w-v\|
$$

where $\gamma=M_{1}+L M$.

Then the equality $v^{*}=w^{*}$ will be held, if $\gamma=M_{1}+L M<1$, and the following theorem holds.

Theorem 3. Let $\left\langle v^{0}, w^{0}>\right.$ be a strongly invariant cone segment for the heterotone operator $T$ of the form (13) with the companion operator $\hat{T}$ of the form (14) and the condition (25) holds, besides, $\gamma=M_{1}+L M<1$, where the constants $M$ and $M_{1}$ are defined by the equalities (24). Then, the iterative process (17)-(19) bilaterally converges in the norm of the space $C(\bar{\Omega})$ to the unique on $\left\langle v^{0}, w^{0}>\right.$ continuous positive solution $u^{*}$ of the boundary value problem (1)-(3).

If the $k$-th iteration have been performed, then as an approximate solution of the boundary value problem (1)-(3) the function

$$
u^{(k)}(\mathbf{x})=\frac{w^{(k)}(\mathbf{x})+v^{(k)}(\mathbf{x})}{2}
$$

is accepted.

Then for an approximate solution (26) there will be convenient a posteriori estimate of the error:

$$
\left\|u^{*}-u^{(k)}\right\| \leq \frac{1}{2} \max _{\mathbf{x} \in \bar{\Omega}}\left(w^{(k)}(\mathbf{x})-v^{(k)}(\mathbf{x})\right) .
$$

The presence of an estimation of the form (27) is an unconditional advantage of the constructed two-sided iterative process. 
If the accuracy $\varepsilon>0$ is given, then the iterative process should be carried out until the inequality

$$
\max _{\mathbf{x} \in \bar{\Omega}}\left(w^{(k)}(\mathbf{x})-v^{(k)}(\mathbf{x})\right)<2 \varepsilon
$$

will be satisfied and then with an accuracy $\varepsilon$ it can be expected that $u^{*}(\mathbf{x}) \approx u^{(k)}(\mathbf{x})$.

If the conditions of Theorem 3 are satisfied, then an a priori estimate of the error will be:

$$
\left\|u^{*}-u^{(k)}\right\| \leq \frac{\gamma^{k}}{2} \max _{\mathbf{x} \in \bar{\Omega}}\left(w^{0}(\mathbf{x})-v^{0}(\mathbf{x})\right) .
$$

Then from the inequality

$$
\frac{\gamma^{k}}{2} \max _{\mathbf{x} \in \bar{\Omega}}\left(w^{0}(\mathbf{x})-v^{0}(\mathbf{x})\right)<\varepsilon
$$

we obtained that to achieve the accuracy $\varepsilon$ it is necessary to do

$$
k_{0}(\varepsilon)=\left[\frac{\ln \frac{\max _{\mathbf{x} \in \bar{\Omega}}\left(w^{0}(\mathbf{x})-v^{0}(\mathbf{x})\right)}{2 \varepsilon}}{\ln \frac{1}{M_{1}+L M}}\right]+1
$$

iterations, where the square brackets denote the integer part of the number.

\section{NUMERICAL EXPERIMENTS}

The construction of two-sided approximations to the solution of the boundary value problem (1)-(3) will be demonstrated on the problem with exponential nonlinearities:

$$
\begin{gathered}
\mathfrak{L} u=e^{u}+2 e^{-u}, \quad \mathbf{x} \in \Omega, \\
u(\mathbf{x})>0, \quad \mathbf{x} \in \Omega, \\
\left.u\right|_{\partial \Omega}=0,
\end{gathered}
$$

where $\mathfrak{L} u \equiv-\Delta u$ or $\mathfrak{L} u \equiv-\Delta u+u, \Omega=\left\{\mathbf{x}=\left(x_{1}, x_{2}\right): 0<x_{1}, x_{2}<1\right\}$.

The function $f(\mathbf{x}, u)=e^{u}+2 e^{-u}$ is positive and continuous with respect to the set of variables, if $u>0$, and it allows a diagonal representation with the help of function

$$
\hat{f}(\mathbf{x}, v, w)=e^{v}+2 e^{-w} .
$$

The problem (29)-(31) is replaced by an equivalent integral equation

$$
u(\mathbf{x})=\int_{\Omega} K_{2}(\mathbf{x}, \mathbf{s}) u(\mathbf{s}) d \mathbf{s}+\int_{\Omega} Q_{2}(\mathbf{x}, \mathbf{s})\left[e^{u(\mathbf{s})}+2 e^{-u(\mathbf{s})}\right] d \mathbf{s},
$$

where $Q_{2}(\mathbf{x}, \mathbf{s})$ is determined by the formula (5), if $\mathfrak{L} u \equiv-\Delta u$, and is determined by the formula (7), if $\mathfrak{L} u \equiv-\Delta u+u, K_{2}(\mathbf{x}, \mathbf{s})=-\frac{\partial^{2}}{\partial s_{1}^{2}} \tilde{g}_{2}(\mathbf{x}, \mathbf{s})-\frac{\partial^{2}}{\partial s_{2}^{2}} \tilde{g}_{2}(\mathbf{x}, \mathbf{s})$,

$$
\begin{gathered}
\tilde{g}_{2}(\mathbf{x}, \mathbf{s})=\frac{1}{2 \pi} \ln \frac{1}{\sqrt{r^{2}+4 \omega(\mathbf{x}) \omega(\mathbf{s})}}, \text { if } \mathfrak{L} u \equiv-\Delta u, \\
\tilde{g}_{2}(\mathbf{x}, \mathbf{s})=\frac{1}{2 \pi} K_{0}\left(\kappa \sqrt{r^{2}+4 \omega(\mathbf{x}) \omega(\mathbf{s})}\right), \text { if } \mathfrak{L} u \equiv-\Delta u+u,
\end{gathered}
$$




$$
\omega(\mathbf{x})=\left[x_{1}\left(1-x_{1}\right)\right] \wedge_{0}\left[x_{2}\left(1-x_{2}\right)\right] \equiv x_{1}\left(1-x_{1}\right)+x_{2}\left(1-x_{2}\right)-\sqrt{x_{1}^{2}\left(1-x_{1}\right)^{2}+x_{2}^{2}\left(1-x_{2}\right)^{2}} .
$$

With the equation (32) let us associate a heterotone operator

$$
T(u)(\mathbf{x})=\int_{\Omega} K_{2}(\mathbf{x}, \mathbf{s}) u(\mathbf{s}) d \mathbf{s}+\int_{\Omega} Q_{2}(\mathbf{x}, \mathbf{s})\left[e^{u(\mathbf{s})}+2 e^{-u(\mathbf{s})}\right] d \mathbf{s},
$$

for which the companion operator has the form

$$
\hat{T}(v, w)(\mathbf{x})=\int_{\Omega} K_{2}^{+}(\mathbf{x}, \mathbf{s}) v(\mathbf{s}) d \mathbf{s}-\int_{\Omega} K_{2}^{-}(\mathbf{x}, \mathbf{s}) w(\mathbf{s}) d \mathbf{s}+\int_{\Omega} Q_{2}(\mathbf{x}, \mathbf{s})\left[e^{v(\mathbf{s})}+2 e^{-w(\mathbf{s})}\right] d \mathbf{s},
$$

where

$$
K_{2}^{+}(\mathbf{x}, \mathbf{s})=\max \left\{0, K_{2}(\mathbf{x}, \mathbf{s})\right\}, \quad K_{2}^{-}(\mathbf{x}, \mathbf{s})=\max \left\{0,-K_{2}(\mathbf{x}, \mathbf{s})\right\} .
$$

For the operator $T$ of the form (33) a strongly invariant cone segment will be sought in the form $<v^{0}, w^{0}>$, where $v^{0}(\mathbf{x})=\alpha u_{0}(\mathbf{x}), w^{0}(\mathbf{x})=\beta u_{0}(\mathbf{x}), 0<\alpha<\beta$, and

$$
u_{0}(\mathbf{x})=\int_{\Omega} Q_{2}(\mathbf{x}, \mathbf{s}) d \mathbf{s} .
$$

For the chosen functions $v^{0}, w^{0}$ the system of inequalities (15), (16) leads to the next system of inequalities for determining the constants $\alpha, \beta$ : for all $\mathbf{x} \in \bar{\Omega}$

$$
\begin{aligned}
& \alpha \int_{\Omega} K_{2}^{+}(\mathbf{x}, \mathbf{s}) u_{0}(\mathbf{s}) d \mathbf{s}-\beta \int_{\Omega} K_{2}^{-}(\mathbf{x}, \mathbf{s}) u_{0}(\mathbf{s}) d \mathbf{s}+\int_{\Omega} Q_{2}(\mathbf{x}, \mathbf{s})\left[e^{\alpha u_{0}(\mathbf{s})}+2 e^{-\beta u_{0}(\mathbf{s})}\right] d \mathbf{s} \geq \alpha u_{0}(\mathbf{x}), \\
& \beta \int_{\Omega} K_{2}^{+}(\mathbf{x}, \mathbf{s}) u_{0}(\mathbf{s}) d \mathbf{s}-\alpha \int_{\Omega} K_{2}^{-}(\mathbf{x}, \mathbf{s}) u_{0}(\mathbf{s}) d \mathbf{s}+\int_{\Omega} Q_{2}(\mathbf{x}, \mathbf{s})\left[e^{\beta u_{0}(\mathbf{s})}+2 e^{-\alpha u_{0}(\mathbf{s})}\right] d \mathbf{s} \leq \beta u_{0}(\mathbf{x}) .
\end{aligned}
$$

If $0<v, w<M_{0}$, where $M_{0}=\beta \max _{x \in \bar{\Omega}} u_{0}(\mathbf{x})$, then

$$
|\hat{f}(\mathbf{x}, v, w)-\hat{f}(\mathbf{x}, w, v)|=\left|\left(e^{v}+2 e^{-w}\right)-\left(e^{w}+2 e^{-v}\right)\right| \leq\left(2+e^{M_{0}}\right)|v-w| .
$$

For the considering problem the iterative process (17)-(19) has the form

$$
\begin{aligned}
& v^{(k+1)}(\mathbf{x})=\int_{\Omega} K_{2}^{+}(\mathbf{x}, \mathbf{s}) v^{(k)}(\mathbf{s}) d \mathbf{s}-\int_{\Omega} K_{2}^{-}(\mathbf{x}, \mathbf{s}) w^{(k)}(\mathbf{s}) d \mathbf{s} \\
&+\int_{\Omega} Q_{2}(\mathbf{x}, \mathbf{s})\left[e^{v^{(k)}(\mathbf{s})}+2 e^{-w^{(k)}(\mathbf{s})}\right] d \mathbf{s} \\
& w^{(k+1)}(\mathbf{x})=\int_{\Omega} K_{2}^{+}(\mathbf{x}, \mathbf{s}) w^{(k)}(\mathbf{s}) d \mathbf{s}-\int_{\Omega} K_{2}^{-}(\mathbf{x}, \mathbf{s}) v^{(k)}(\mathbf{s}) d \mathbf{s} \\
&+\int_{\Omega} Q_{2}(\mathbf{x}, \mathbf{s})\left[e^{w^{(k)}(\mathbf{s})}+2 e^{-v^{(k)}(\mathbf{s})}\right] d \mathbf{s}, \\
& k=0,1,2, \ldots, \\
& v^{(0)}(\mathbf{x})=\alpha u_{0}(\mathbf{x}), \quad w^{(0)}(\mathbf{x})=\beta u_{0}(\mathbf{x}) .
\end{aligned}
$$


For the problem (29)-(31), if $\mathfrak{L} u \equiv-\Delta u$, it was found, that the system of inequalities for determining the constants $\alpha, \beta$ is satisfied by the values $\alpha=2.5, \beta=5.8$. Further we find

$$
\begin{gathered}
M=\max _{\mathbf{x} \in \bar{\Omega}} \int_{\Omega} Q_{2}(\mathbf{x}, \mathbf{s}) d \mathbf{s}=0.04093, \quad M_{1}=\max _{\mathbf{x} \in \bar{\Omega}} \int_{\Omega}\left[K_{2}^{+}(\mathbf{x}, \mathbf{s})+K_{2}^{-}(\mathbf{x}, \mathbf{s})\right] d \mathbf{s}=0.70819, \\
M_{0}=\beta_{x \in \bar{\Omega}} \max _{0}(\mathbf{x})=0.23740, \quad L=2+e^{M_{0}}=3.26795, \quad \gamma=M_{1}+L M=0.842 .
\end{gathered}
$$

Thus, $\gamma<1$ and by Theorem 3, the successive approximations that are formed by the scheme (34)-(36) bilaterally converge to the solution of the problem (29)-(31) for $\mathfrak{L} u \equiv-\Delta u$.

Let us choose $\varepsilon=10^{-4}$. Then, in accordance with (28), to achieve this accuracy, it is necessary to make $k_{0}(\varepsilon)=\left[\frac{\ln \frac{(\beta-\alpha) M}{2 \varepsilon}}{\ln \frac{1}{\gamma}}\right]+1=38$ iterations. In fact, the accuracy $\varepsilon=10^{-4}$ was achieved at the eleventh iteration. As one can see, the theoretical error estimate turned out to be greatly overestimated. As an approximate solution of the problem (29)-(31) for $\mathfrak{L} u \equiv-\Delta u$, the function $u^{(11)}(\mathbf{x})=\frac{v^{(11)}(\mathbf{x})+w^{(11)}(\mathbf{x})}{2}$ will be accepted.

\begin{tabular}{|c|c|c|c|c|c|c|}
\hline $\begin{array}{c}\text { Iteration } \\
\text { number } k\end{array}$ & 0 & 1 & 2 & 3 & 4 & 5 \\
\hline$\varepsilon^{(k)}$ & $0.67 \cdot 10^{-1}$ & $0.39 \cdot 10^{-1}$ & $0.22 \cdot 10^{-1}$ & $0.22 \cdot 10^{-1}$ & $0.65 \cdot 10^{-2}$ & $0.35 \cdot 10^{-2}$ \\
\hline $\begin{array}{c}\text { Iteration } \\
\text { number } k\end{array}$ & 6 & 7 & 8 & 9 & 10 & 11 \\
\hline$\varepsilon^{(k)}$ & $0.19 \cdot 10^{-2}$ & $0.10 \cdot 10^{-2}$ & $0.57 \cdot 10^{-3}$ & $0.31 \cdot 10^{-3}$ & $0.17 \cdot 10^{-3}$ & $0.91 \cdot 10^{-4}$ \\
\hline
\end{tabular}

Table 1 . The values of the estimate $\varepsilon^{(k)}$ of the approximate solution error for $\mathfrak{L} u \equiv-\Delta u$

Table 1 gives the data how the estimate $\varepsilon^{(k)}=\max _{\mathbf{x} \in \bar{\Omega}} \frac{1}{2}\left(w^{(k)}(\mathbf{x})-v^{(k)}(\mathbf{x})\right)$ of the norm of the error $\left\|u^{*}-u^{(k)}\right\|$ of the approximate solution $u^{(k)}(\mathbf{x})$ varies depending on the iteration number $k, k=0,1, \ldots, 11$. It was found that $\left\|u^{(11)}\right\|=0.2130$.

Figure 1 shows the graph of the cross-sections of the upper $w^{(k)}(\mathbf{x})$ and the lower $v^{(k)}(\mathbf{x})$ approximations at $x_{2}=0.5$ for $k=0,2,6,8$. Figures 2,3 show the surface of the approximate solution $u^{(11)}(\mathbf{x})$ and its contour lines (with the step 0.02) respectively. Considering the relationship $\frac{\varepsilon^{(k+1)}}{\varepsilon^{(k)}}, k=0,1, \ldots, 11$, according to the Table 1 , it was received that $\frac{\varepsilon^{(k+1)}}{\varepsilon^{(k)}} \approx 0.543$, that indicates the geometric rate of convergence of the iterative sequence with the corresponding index. Let us note that the convergence exponent turned out to be less than the exponent $\gamma$ estimated in accordance with Theorem 3.

Let us now consider the problem (29)-(31) for $\mathfrak{L} u \equiv-\Delta u+u$. It was found that the system of inequalities to determine the constants $\alpha, \beta$ is satisfied by the values $\alpha=0.1, \beta=5.3$. Further we find

$$
\begin{gathered}
M=\max _{\mathbf{x} \in \bar{\Omega}} \int_{\Omega} Q_{2}(\mathbf{x}, \mathbf{s}) d \mathbf{s}=0.03760, \\
M_{1}=\max _{\mathbf{x} \in \bar{\Omega}} \int_{\Omega}\left[K_{2}^{+}(\mathbf{x}, \mathbf{s})+K_{2}^{-}(\mathbf{x}, \mathbf{s})\right] d \mathbf{s}=0.60410, \\
M_{0}=\beta \max _{x \in \bar{\Omega}} u_{0}(\mathbf{x})=0.19929, \quad L=2+e^{M_{0}}=3.22053, \quad \gamma=M_{1}+L M=0.725 .
\end{gathered}
$$


Thus, $\gamma<1$ and by Theorem 3, the successive approximations that are formed by the scheme (34)-(36) bilaterally converge to the solution of the problem (29)-(31) for $\mathfrak{L} u \equiv-\Delta u+u$.

Let us choose $\varepsilon=10^{-4}$. Then, in accordance with (28), to achieve this accuracy, it is necessary to make $k_{0}(\varepsilon)=\left[\frac{\ln \frac{(\beta-\alpha) M}{2 \varepsilon}}{\ln \frac{1}{\gamma}}\right]+1=22$ iterations. In fact, the accuracy $\varepsilon=10^{-4}$ was achieved at the tenth iteration. As one can see, the theoretical error estimate turned out to be greatly overestimated too. As an approximate solution of the problem (29)-(31) for $\mathfrak{L} u \equiv-\Delta u+u$, the function $u^{(10)}(\mathbf{x})=\frac{v^{(10)}(\mathbf{x})+w^{(10)}(\mathbf{x})}{2}$ will be accepted.

\begin{tabular}{|c|c|c|c|c|c|c|}
\hline $\begin{array}{c}\text { Iteration } \\
\text { number } k\end{array}$ & 0 & 1 & 2 & 3 & 4 & 5 \\
\hline$\varepsilon^{(k)}$ & $0.98 \cdot 10^{-1}$ & $0.49 \cdot 10^{-1}$ & $0.24 \cdot 10^{-1}$ & $0.12 \cdot 10^{-1}$ & $0.58 \cdot 10^{-2}$ & $0.28 \cdot 10^{-2}$ \\
\hline $\begin{array}{c}\text { Iteration } \\
\text { number } k\end{array}$ & 6 & 7 & 8 & 9 & 10 & \\
\hline$\varepsilon^{(k)}$ & $0.14 \cdot 10^{-2}$ & $0.67 \cdot 10^{-3}$ & $0.33 \cdot 10^{-3}$ & $0.16 \cdot 10^{-3}$ & $0.78 \cdot 10^{-4}$ & \\
\hline
\end{tabular}

Table 2. The values of the estimate $\varepsilon^{(k)}$ of the approximate solution error for $\mathfrak{L} u \equiv-\Delta u+u$

Table 2 gives the data how the estimate $\varepsilon^{(k)}=\max _{\mathbf{x} \in \bar{\Omega}} \frac{1}{2}\left(w^{(k)}(\mathbf{x})-v^{(k)}(\mathbf{x})\right)$ of the norm of the error $\left\|u^{*}-u^{(k)}\right\|$ of the approximate solution $u^{(k)}(\mathbf{x})$ varies depending on the iteration number $k, k=0,1, \ldots, 10$. It was found that $\left\|u^{(10)}\right\|=0.1742$. We can see, the norm of the approximate solution in the transition to the equation with $\mathfrak{L} u \equiv-\Delta u+u$ has decreased.

Figure 4 shows the graph of the cross-sections of the upper $w^{(k)}(\mathbf{x})$ and the lower $v^{(k)}(\mathbf{x})$ approximations at $x_{2}=0.5$ for $k=0,2,6,8$. Figures 5,6 show the surface of the approximate solution $u^{(10)}(\mathbf{x})$ and its contour lines (with the step 0.02$)$ respectively. Considering the relationship $\frac{\varepsilon^{(k+1)}}{\varepsilon^{(k)}}, k=0,1, \ldots, 10$, according to the Table 2 , it was received that $\frac{\varepsilon^{(k+1)}}{\varepsilon^{(k)}} \approx 0.488$, that indicates the geometric rate of convergence of the iterative sequence with the corresponding index. Let us note that for case $\mathfrak{L} u \equiv-\Delta u+u$ the convergence exponent turned out to be less than the exponent $\gamma$ estimated in accordance with Theorem 3.

\section{CONCLUSIONS}

In the paper a method of two-sided approximations of the solution of the homogeneous Dirichlet problem for a semilinear elliptic equation with Laplace operator $-\Delta$ and Helmholtz operator $-\Delta+\kappa^{2}$ is proposed on the basis of the Green-Rvachev's quasi-function method. A computational experiment carried out for two equations with heterotone exponential nonlinearity demonstrated the possibilities and effectiveness of the method. The proposed approach to the numerical solution of semilinear elliptic equations can be used in solving various applied problems, the mathematical model of which is the problem (1)-(3). The proposed method is more universal than the existing methods, and it allows to solve the boundary problem in domains of arbitrary geometry, provided that this domain can be described by the R-function method. 


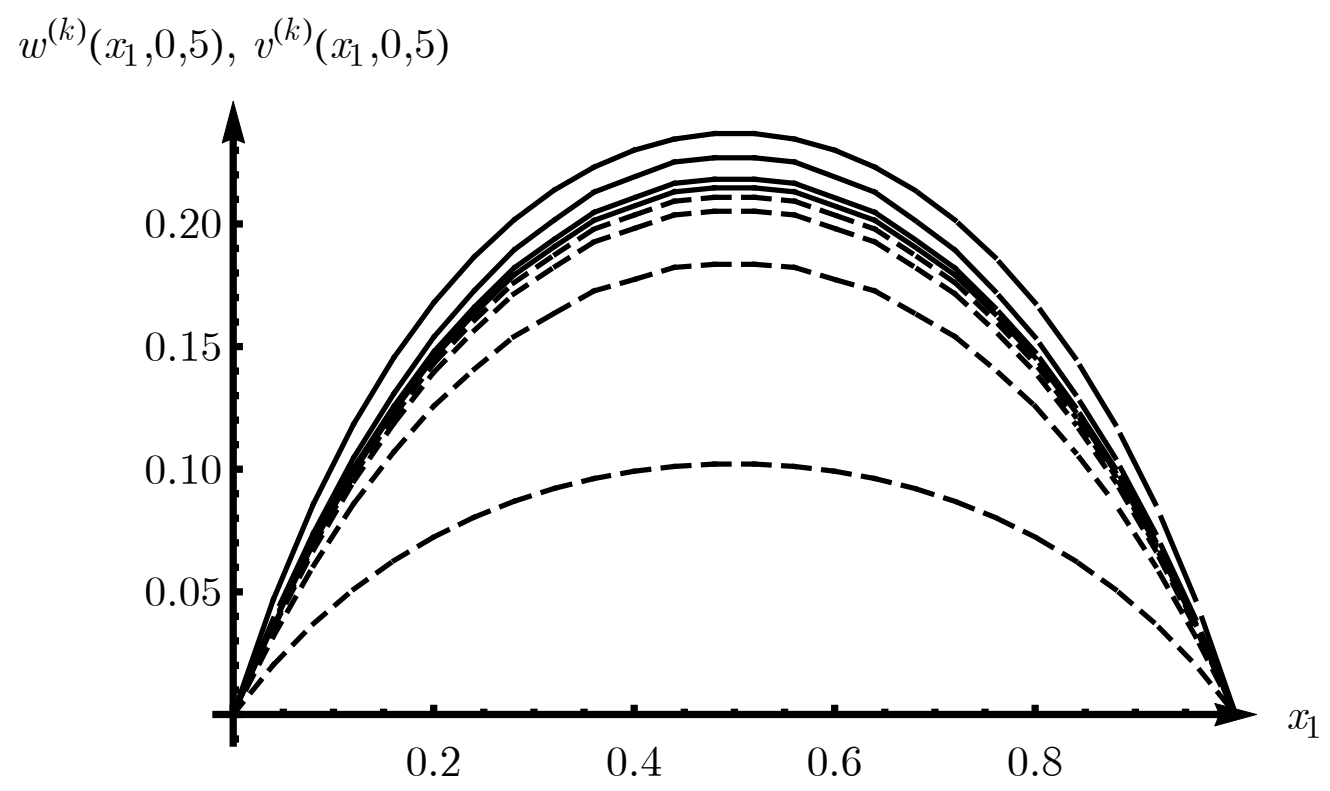

Figure 1. Graph of the cross-sections of upper and lower approximations $w^{(k)}\left(x_{1}, 0.5\right), v^{(k)}\left(x_{1}, 0.5\right), k=0,2,6,8$, for $\mathfrak{L} u \equiv-\Delta u$

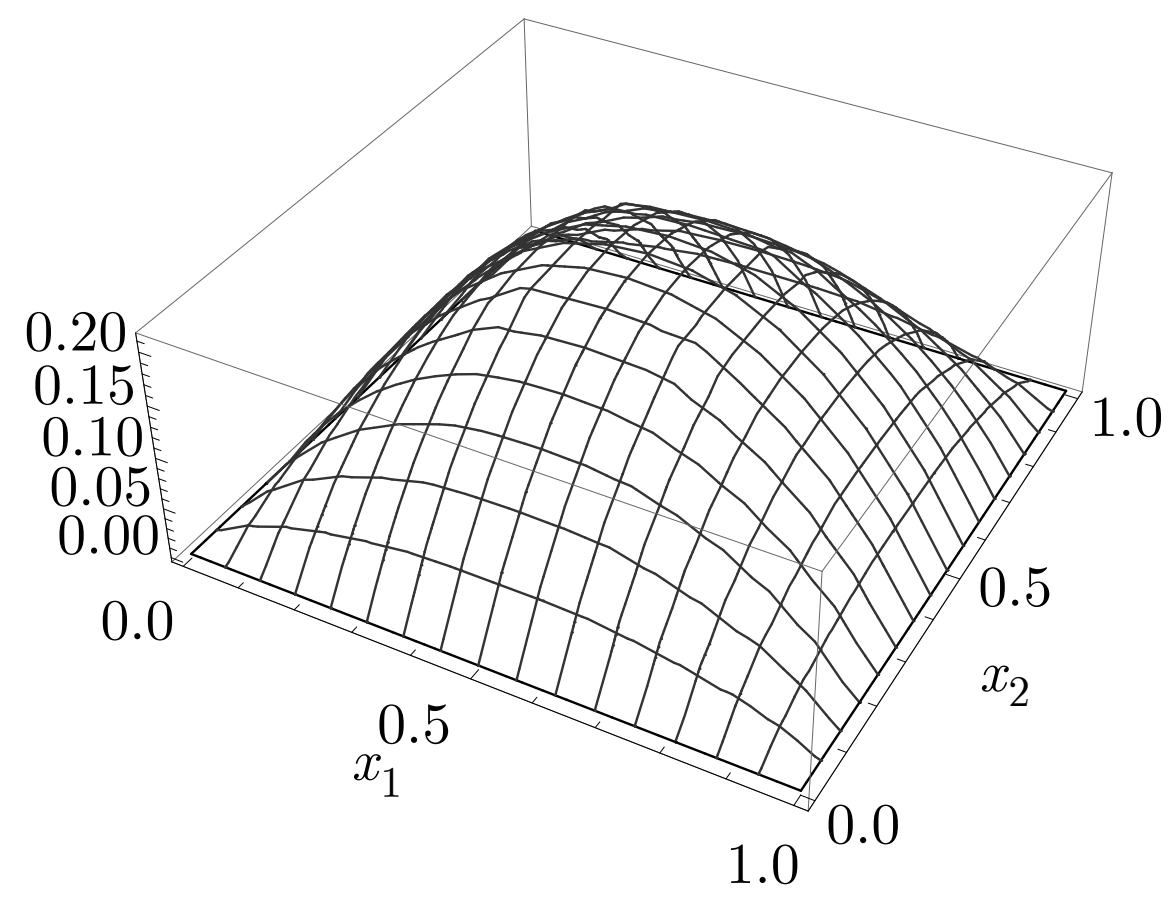

Figure 2. Surface of the approximate solution $u^{(11)}(\mathbf{x})$ for $\mathfrak{L} u \equiv-\Delta u$ 


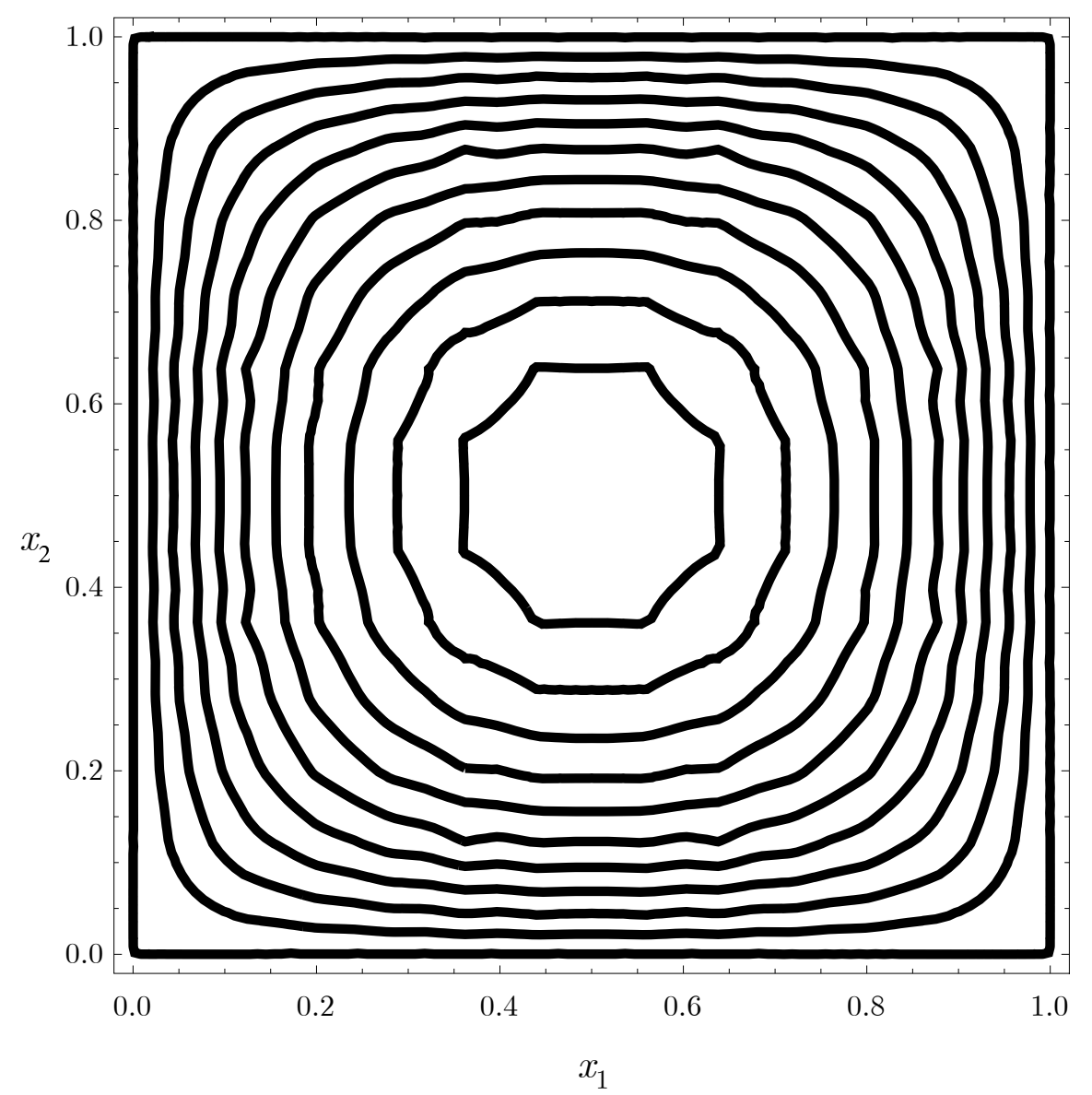

Figure 3. Contour lines of the approximate solution $u^{(11)}(\mathbf{x})$ for $\mathfrak{L} u \equiv-\Delta u$ $w^{(k)}\left(x_{1}, 0,5\right), v^{(k)}\left(x_{1}, 0,5\right)$

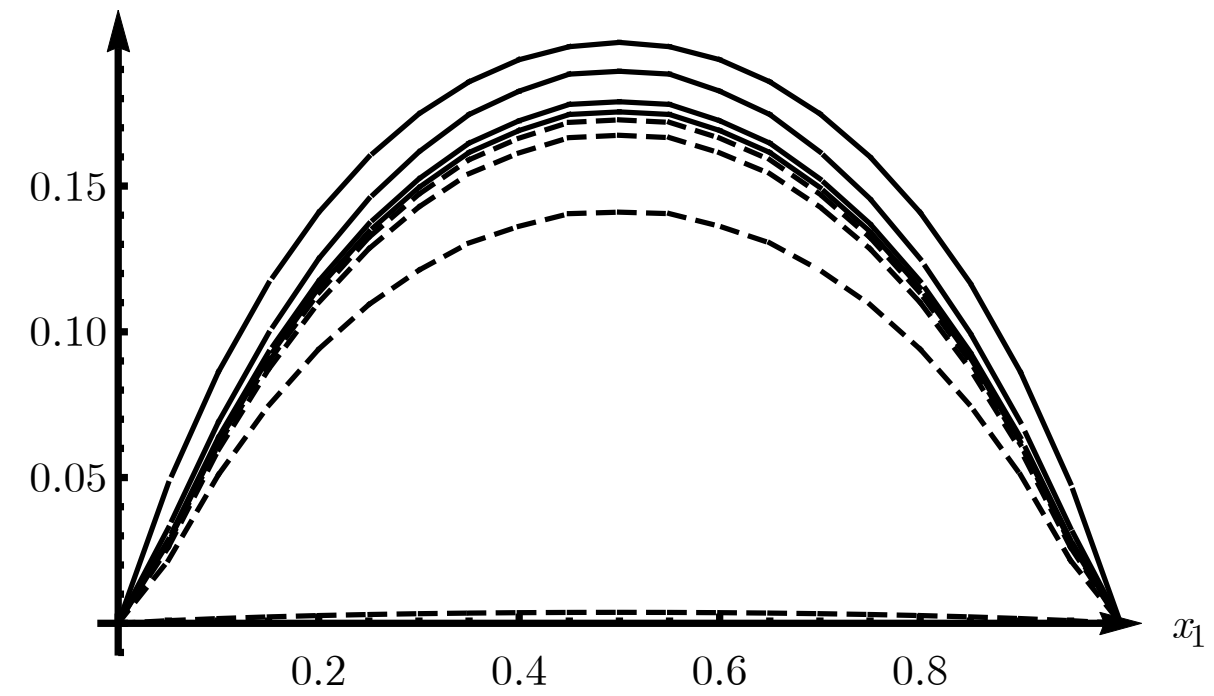

Figure 4. Graph of the cross-sections of upper and lower approximations $w^{(k)}\left(x_{1}, 0.5\right), v^{(k)}\left(x_{1}, 0.5\right), k=0,2,6,8$, for $\mathfrak{L} u \equiv-\Delta u+u$ 


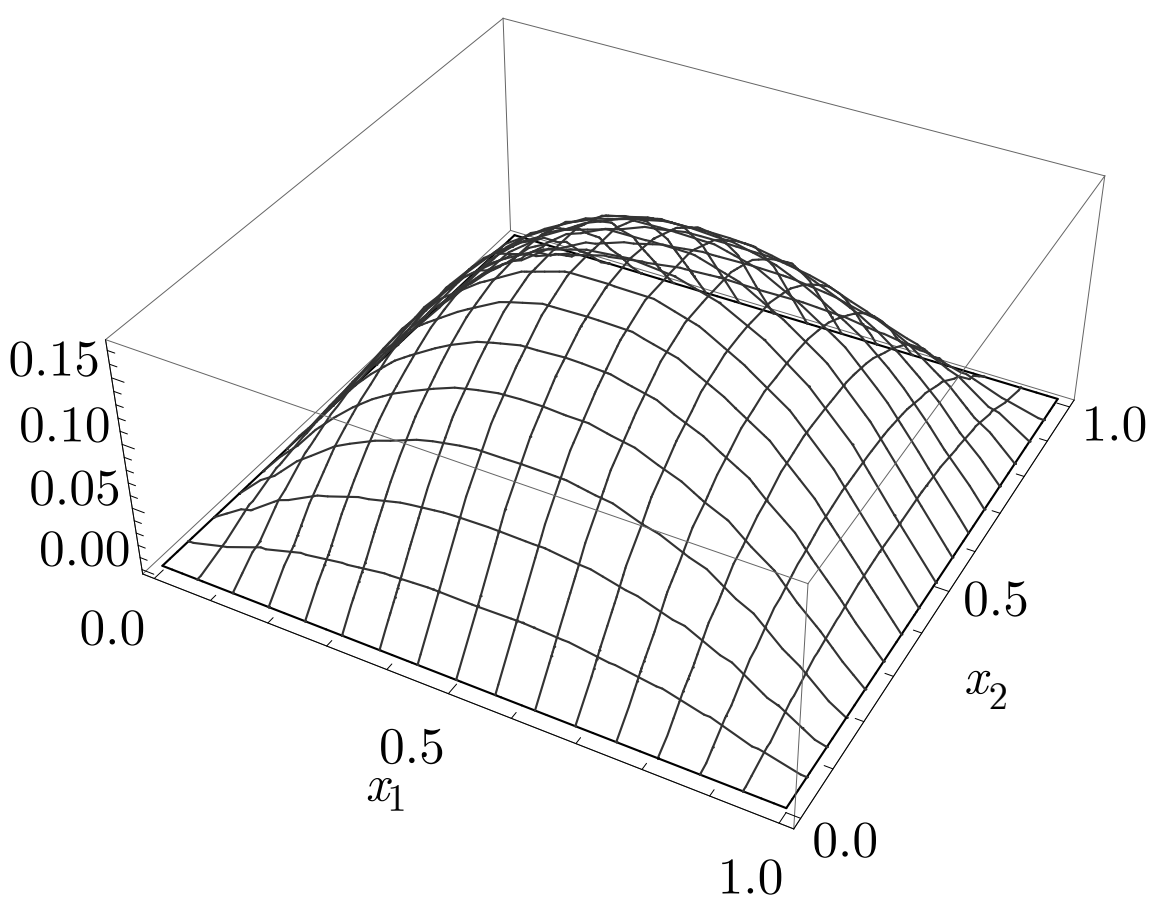

Figure 5. Surface of the approximate solution $u^{(10)}(\mathbf{x})$ for $\mathfrak{L} u \equiv-\Delta u+u$

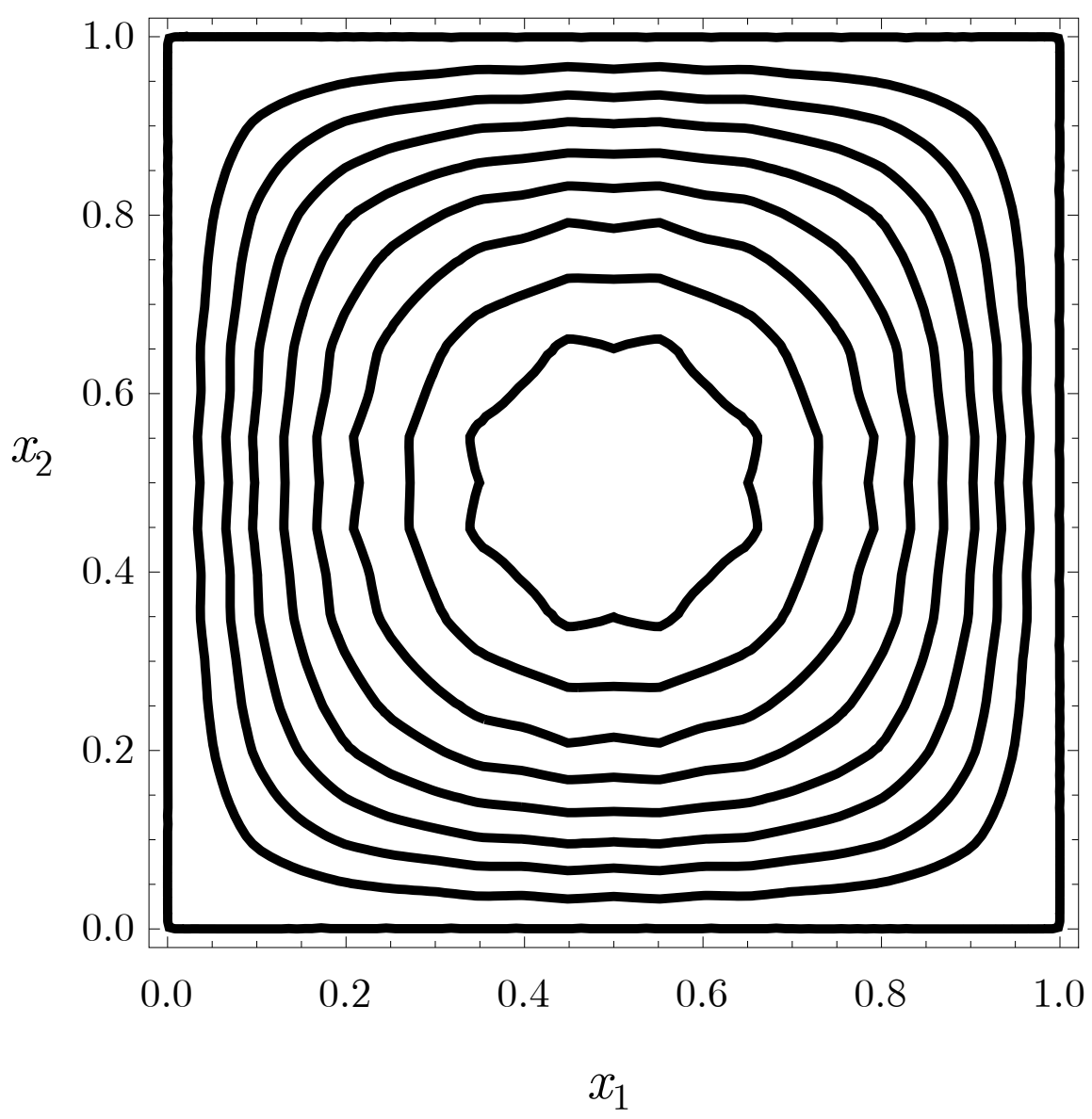

Figure 6. Contour lines of the approximate solution $u^{(10)}(\mathbf{x})$ for $\mathfrak{L} u \equiv-\Delta u+u$ 


\section{REFERENCES}

[1] Amann H. Fixed point equations and nonlinear eigenvalue problems in ordered Banach spaces. SIAM Review 1976, 18 (4), 620-709. doi:10.1137/1018114

[2] Guo D., Lakshmikantham V. Coupled fixed points of nonlinear operators with applications. Nonlinear Anal. 1987, 11 (5), 623-632. doi:10.1016/0362-546X(87)90077-0

[3] Kolosov A.I. A class of boundary value problems reducible to an equation with a heterotonic operator. Differ. Uravn. 1985, 21 (11), 1884-1891. (in Russian)

[4] Kolosova S.V., Lukhanin V.S., Sidorov M.V. On the construction of two-sided approximations to the positive solution of the Lane-Emden equation. Visn. of Zaporizh. Nat. Univ. Phys. and Math. Sc. 2015, 3, 107-120. (in Russian)

[5] Krasnosel'skii M.A. Positive solutions of operator equations. Fizmatgiz, Moscow, 1962. (in Russian)

[6] Kurpel' N.S., Shuvar B.A. Two-sided operator inequalities and its applications. Naukova Dumka, Kyiv, 1980. (in Russian)

[7] Mikhlin S.G. A course in mathematical physics. Nauka, Moscow, 1968. (in Russian)

[8] Miranda C. Partial Differential Equations of Elliptic Type. Springer, Berlin, 1970. doi:10.1007/978-3-642$87773-5$

[9] Opoǐcev V.I. Generalization of the theory of monotone and concave operators. Tr. Mosk. Mat. Obs. 1978, 36, $237-273$.

[10] Opoǐcev V.I., Khurodze T.A. Nonlinear operators in spaces with a cone. Izdatel'stvo Tbilisskogo Universiteta, Tbilisi, 1984. (in Russian)

[11] Pao C.V. Nonlinear parabolic and elliptic equations. Plenum Press, New York, 1992.

[12] Rvachev V.L. Theory of R-functions and some of its applications. Naukova Dumka, Kiev, 1982. (in Russian)

[13] Shuvar B.A., Kopach M.I., Mentynskyy S.M., Obshta A.F. Two-sided approximate methods. VDV CIT, IvanoFrankivs'k, 2007. (in Ukrainian)

[14] Sidorov M.V. Construction two-sided iterative processes for solving nonlinear boundary value problems using methods of Green's functions and the quasi-functions of Green-Rvachev. Visn. of Zaporizh. Nat. Univ. Phys. and Math. Sc. 2017, 2, 250-259. (in Ukrainian)

Received 09.05.2018

Силоров М.В. Метод квазіфункиій Гріна-Рвачова побудови двобічних наближень до додатного розв'язку нелінійних крайових задач // Карпатські матем. публ. - 2018. - Т.10, №2. - С. 360-375.

Розглядається однорідна задача Аіріхле для напівлінійних еліптичних рівнянь з оператором Аапласа та оператором Гельмгольця. Аля побудови двобічних наближень до додатного розв'язку цієї крайової задачі використовується перехід за допомогою квазіфункції ГрінаРвачова до еквівалентного нелінійного інтегрального рівняння з подальшим його аналізом методами теорії напівупорядкованих просторів. Робота і ефективність розробленого метода продемонстрована обчислювальним експериментом для тестової задачі з експоненціальною нелінійністю.

Ключові слова і фрази: додатний розв'язок, напівлінійна еліптична крайова задача, гетеротонний оператор, двобічні наближення, квазіфункція Гріна-Рвачова. 